\title{
Polypropylene Carbon Nanotubes Nanocomposites: Combined Influence of Block Copolymer Compatibilizer and Melt Annealing on Electrical Properties
}

\author{
Aline Emplit, ${ }^{1}$ Fang Fang Tao, ${ }^{2}$ Pascale Lipnik, ${ }^{2}$ Guido Heunen, ${ }^{2}$ \\ Christian Bailly, ${ }^{2}$ and Isabelle Huynen ${ }^{1}$ \\ ${ }^{1}$ Information and Communications Technologies, Electronics and Applied Mathematics (ICTEAM), Université Catholique de Louvain, \\ Place du Levant 3, 1348 Louvain-la-Neuve, Belgium \\ ${ }^{2}$ Institute of Condensed Matter and Nanosciences (IMCN), Université Catholique de Louvain, Croix du Sud 1, \\ 1348 Louvain-la-Neuve, Belgium
}

Correspondence should be addressed to Isabelle Huynen; isabelle.huynen@uclouvain.be

Received 9 March 2017; Accepted 24 May 2017; Published 4 July 2017

Academic Editor: Bhanu P. Singh

Copyright (C) 2017 Aline Emplit et al. This is an open access article distributed under the Creative Commons Attribution License, which permits unrestricted use, distribution, and reproduction in any medium, provided the original work is properly cited.

\begin{abstract}
We study the influence of melt annealing and the presence of a block copolymer compatibilizer on the electrical properties of polypropylene carbon nanotubes (CNT) nanocomposites from the DC limit to microwave frequencies and link it to the morphological details. We show that the compatibilizer concentration controls three types of morphologies: separate CNT agglomerates, a network of well dispersed but interconnected CNT, and individualized but separate nanotubes. This explains why conductivity reaches an optimum over the whole frequency range at a low compatibilizer concentration. We model the corresponding structures by a semiquantitative schematic equivalent electrical circuit. A key outcome of the work is the understanding and control of dispersion mechanisms in order to optimize the electrical performances for efficient EMI shielding depending on the targeted frequency range.
\end{abstract}

\section{Introduction}

The ever expanding use of electrically insulating polymers as enclosure materials for electrical, electronic, and high frequency (HF) wireless communication devices exacerbates electrostatic dissipation and electromagnetic interference (EMI) issues but at the same time opens up new opportunities for original solutions.

For bulk or surface DC conductivity, the main challenge is to conceive materials that retain high mechanical performance combined with adequate electrical conductivity. This naturally leads to (nano)composites of a dielectric matrix and a well dispersed conductive filler, preferably with high aspect ratio, providing electrical percolation at low concentration. Polymer-carbon nanotubes (CNT) nanocomposites are clearly attractive from that standpoint if dispersion can be controlled [1-3]. It is important to realize that the contact resistance between $\mathrm{CNT}$ dominates the conductivity of percolation networks in DC and low frequency regimes. This implies that while optimal dispersion of the CNT is favorable for conductivity, the contact distance and resistance between them must be minimized to allow conduction by electron hopping [4-7]. Insulating irreversible coating on the $\mathrm{CNT}$ is hence detrimental.

EMI shielding is nowadays a very challenging issue because electronic devices are becoming ever more compact and integrated, which increases spurious coupling interferences either by direct transmission or through multiple reflections. This leads to a renewed interest for electromagnetic absorber materials, which simultaneously reduce the reflection and transmission of the signal. Besides metamaterials designed to absorb specific frequencies by resonance [8], composite-based materials containing conductive inclusions, for example, metal powders, ferrites, carbon, and conjugated polymers, have already been proposed as electromagnetic 
(EM) absorbers [9-19]. Again, well dispersed polymerCNT nanocomposites (possibly foamed, see $[20,21]$ ) are attractive for broadband absorption in the $\mathrm{GHz}$ frequency range because the high aspect ratio of CNT allows reaching sufficient conductivity at low filler content and hence the elusive combination of high conductivity and low dielectric constant, which is essential for effective absorption $[5,21-$ 24]. HF conductivity of polymer composites follows different rules than DC conductivity. Above a critical frequency, electron hopping is no longer the dominant mechanism but conductivity results from Resistive-Capacitive (RC) coupling between filler and matrix, which can be described by equivalent circuits [5, 25-27]. EMI shielding CNT-based polymer nanocomposites based on, for example, polycarbonate, polycaprolactone, polypropylene and polystyrene have already been described [20, 21, 28-30]. They still mostly work by reflection, not absorption. Recently, effective EM absorbing structures based on polymer-CNT nanocomposites have been described [21, 31-33].

Polyolefin-CNT nanocomposites, either polyethylene or polypropylene (PP) based, have attracted a lot of academic and industrial interest due to their unique combination of cost effectiveness and properties [21, 24, 34-36]. However, the dispersion of pristine CNT in polyolefins by melt-mixing is usually poor, due to unfavorable interactions between the polymer chains and the CNT as well as strong Van der Waals attraction between the CNT themselves $[37,38]$. This is particularly true for PP, often resulting in poor conductivity and mechanical properties of the composites. To ensure a good load transfer between the CNT and the polymer matrix as well as a good dispersion of the nanofiller, CNT modifications by organic molecules, including polymer chains, can be used. For example, some authors have proposed a covalent modification route to help CNT dispersion in PP $[21,36,39,40]$. However, grafting techniques have the disadvantage of being cumbersome and detrimental to true electrical contacts. Physical coating of the CNT by selective adsorption is a preferable strategy for several reasons: first, it preserves all intrinsic features of the nanotubes; second, it provides efficient surface modification with ease and cost effectiveness; third, it is potentially reversible, which can be of importance to reduce contact resistance between the CNT. The effectiveness of this selective adsorption strategy for improved CNT dispersion or phase/interfacial selectivity has been demonstrated by our research team for acrylateethylene copolymers, polyamides, and polyethylene already [37, 41-43]. One key result of these studies is that the melting sequence of the polymers plays an important role for the final dispersion and morphology: the first polymer to contact the nanotubes during melting has the opportunity to strongly adsorb by noncovalent interaction. Overall, the control of DC as well as HF conductivity of CNT-polymer nanocomposites results from a complex balance between CNT dispersion and electrical networking. At low frequency, the contact resistance between the percolating CNT is the critical parameter while, at high frequency, the details of the morphology control the RC coupling. It is known that melt annealing can allow electrical contacts between the CNT to strengthen under the influence of their mutual Van der Waals attraction
$[37,38]$. This is called secondary agglomeration. However, the mutual attraction between CNT will be stronger in typical situations where they are difficult to disperse (high value of the Hamaker constant). At the opposite, when CNT disperse effectively in a polymer matrix, it is often because their surface has been modified with an insulating organic coating, which is detrimental to electrical contacts. Very few studies have been devoted to a systematic analysis of the combined influence of CNT-polymer compatibility and melt annealing on DC and HF conductivity. On the other hand, there is little literature about composites for absorption below $1 \mathrm{GHz}$; EMI shielding was observed around $200 \mathrm{MHz}$ using carbon nanotubes in poly-methyl-methacrylate [44]. Up to $30 \mathrm{~dB}$ of shielding was obtained. However these performances result from a combination of reflection and absorption rather than pure absorption. Regarding polypropylene composites interesting properties were observed at $800 \mathrm{MHz}$ in [45]. In [46] up to $15 \mathrm{~dB}$ shielding effectiveness was obtained with polypropylene loaded with carbon fibers. In [47] $25 \mathrm{~dB}$ shielding efficiency was obtained; absorption was observed at X-band in polypropylene foams loaded with carbon fibers. Carbon nanotubes are also efficient for absorption above $1 \mathrm{GHz}$. Indeed their high aspect ratio promotes the formation of a conductive path at a low content [33]. Mechanical behavior of absorbers is also of prime interest in airborne applications. Therefore some solutions were investigated combining CNT composite and honeycomb metallic matrix in order to improve resistance to impact [48].

In this work, we use block copolymer compatibilizer (hydrogenated styrene-isoprene-styrene triblock: SEPS) combined with melt annealing to modify the dispersion and electrical contacts of pristine multiwall CNT in a PP matrix. The styrene blocks of SEPS have an affinity for CNT because of their polarisability and potential for pi-pi stacking. The EP blocks on the other hand have an affinity for the PP phase. SEPS block copolymers can hence act as effective compatibilizers, as will be demonstrated below. Varying the SEPS concentration at constant CNT load allows us to systematically vary the nanotubes dispersion, mutual attraction in the matrix, and the resulting electrical properties. We also study the influence of annealing the nanocomposites in the melt on their DC and HF conductivity. The electrical properties are studied over a broad frequency range thanks to the combination of two different techniques, operating in the $0.01 \mathrm{~Hz}$ to $1 \mathrm{MHz}$ and $1 \mathrm{GHz}$ to $67 \mathrm{GHz}$ ranges, respectively. A key outcome of this work is the understanding and control of dispersion mechanisms in order to optimize electrical performances for efficient EMI shielding, depending on the targeted frequency range.

\section{Experimental Part}

2.1. Raw Materials. The CNT used in this study are provided by the company FutureCarbon. They are homogeneous multiwall tubes obtained by the CVD method, with 10-20 concentric graphene layers and an average diameter of $\sim 20 \mathrm{~nm}$. Purity is $98 \mathrm{w} / \mathrm{w} \%$. SEPS copolymer Hybrar 7125 is produced by Kuraray Co. Ltd by hydrogenation of the corresponding 
TABLE 1: Compounded samples compositions and nomenclature.

\begin{tabular}{lcc}
\hline Nomenclature \% wt & PP (w/w \%) & SEPS (w/w \%) \\
\hline PP98 & 98 & 0 \\
PP93 & 93 & 5 \\
PP50 & 50 & 48 \\
PP0 & 0 & 98 \\
\hline
\end{tabular}

SIS copolymer. It has a high vinyl content (1-2 addition of the isoprene monomer during anionic polymerization of the isoprene block is favored over 1-4 addition) and has a melt flow rate of $4 \mathrm{~g} / 10 \mathrm{~min}$ at $230^{\circ} \mathrm{C}$ under $2.16 \mathrm{~kg}$ load. Isotactic polypropylene HF420FB is purchased from Borealis. It has a melting point of $161^{\circ} \mathrm{C}$ and a melt flow rate of $19 \mathrm{~g} / 10 \mathrm{~min}$ at $230^{\circ} \mathrm{C}$ under $2.16 \mathrm{~kg}$ load.

\subsection{Blending and Compression Molding. A twin-screw mini-} compounder (DSM Microextruder $15 \mathrm{~cm}^{3}$ ) is used to compound the nanocomposites. For all melt blending experiments, the conditions are as follows: $250 \mathrm{rpm}, 10$ minutes compounding at $220^{\circ} \mathrm{C}$. All components of the blends are added simultaneously in the hopper after dry-mixing. For further characterization of electrical properties, the compounded samples are compression-molded at $190^{\circ} \mathrm{C}$ for 1,10 , and $20 \mathrm{~min}$, respectively, in a Fontijne press followed by quick cooling.

2.3. Nanocomposite Samples. All nanocomposite samples contain $2 \mathrm{wt} \% \mathrm{CNT}$. In order to enable an exhaustive analysis of the correlation between the structure and electrical properties, we systematically vary the PP/SEPS weight ratio of the polymer matrix. The composition and nomenclature of the samples (except for the pure matrix references) are reported in Table 1. For the same composition, annealing time can range from 1 to $20 \mathrm{~min}$.

\section{Characterization Techniques}

3.1. Transmission Electron Microscopy (TEM). The specimens are cut with the help of a Reichert Microtome. Ultrathin sections of approximately $70-100 \mathrm{~nm}$ in thickness are cut using a cryodiamond knife (Diatome, Switzerland) and collected on 400 mesh copper grids. CNT dispersion in the nanocomposites is investigated with the help of a LEO 922 Transmission Electron Microscope operating at $200 \mathrm{kV}$ or a FEI Tecnai 12 operating at $120 \mathrm{kV}$. To increase contrast between the PP and SEPS phases in blends, some ultrathin sections are exposed to $\mathrm{RuO} 4$ vapor for $30 \mathrm{~min}$ or to $\mathrm{OsO} 4$ for $2 \mathrm{~min}$. The styrene blocks are selectively stained by $\mathrm{RuO} 4$ and thus show up as darker phases on the TEM images. The $\mathrm{OsO} 4$ vapor reacts to stain the residual carbon-carbon double bonds present in SEPS.

3.2. Electrical Measurements. For the frequency range between $0.01 \mathrm{~Hz}$ and $1 \mathrm{MHz}$, a Novocontrol Alpha-A High Performance Frequency Analyser with a Agilent Dielectric Test Fixture is used. The samples are placed between two electrodes to measure the resistance and capacitance, using a parallel equivalent circuit for modeling the behavior.

The relative dielectric constant $\varepsilon_{r}$ and electrical conductivity $\sigma$ of the sample under study are obtained from the measured capacitance $C$ and resistance $R$ according to the expressions $\varepsilon_{r}=\left(C / \varepsilon_{o}\right) \cdot(d / A)$ and $\sigma=d /(R \cdot A)$, where $A$ is the sample area, $d$ the sample thickness, and $\varepsilon_{o}$ the permittivity of vacuum.

For higher frequencies (between $1 \mathrm{GHz}$ and $67 \mathrm{GHz}$ ), a Vector Network Analyser (model N5247A PNA-X Network Analyser from Agilent) is used with a Anristu Model $3680 \mathrm{~V}$ test fixture in order to measure the $S$ parameters of the microstrip line configuration and extract the dielectric constant and electrical conductivity of the sample, according to the method described in [48].

\section{Results}

In nanocomposites, the electrical properties are highly correlated to the nanostructure of the CNT network. Indeed, dispersing conductive nanoparticles in a polymer matrix results in a modification of the dielectric constant and electrical conductivity of the resulting composite. A fine tuning of these parameters is required to maximize absorption of electrical signals in the composite, resulting in improved EMI shielding. In this work, TEM imaging (effective at the nm$\mu \mathrm{m}$ scale) and electrical measurements (which probe the structure at the mm-cm scale) are used as complementary techniques to characterize the dispersion of $\mathrm{CNT}$ in the matrices.

4.1. Morphology of PP/SEPS/CNT Nanocomposites. Figures 1-6 present TEM pictures of the nanocomposites tested in this work. They show both the quality of the CNT dispersion and the morphology of the PP-SEPS mixtures. Most images correspond to composites annealed for 10 minutes in the melt during compression molding of the previously compounded samples (see Experimental Part), after OsO4 or $\mathrm{RuO} 4$ staining. Exceptions are indicated in the caption of the figures. Except for samples with no or low amount of SEPS, which are not affected by annealing time (example in Figure 1), the morphology of the blends evolves upon annealing time until a stable and reproducible structure is observed after approximately $10 \mathrm{~min}$, as can be inferred from the absence of significant evolution between 10 and $20 \mathrm{~min}$ (example in Figure 2). Melt annealing for $10 \mathrm{~min}$ hence allows sufficient macromolecular interdiffusion to reach overall morphological and compositional homogeneity. Images of samples annealed for only $1 \mathrm{~min}$ are shown only for samples containing no or limited to small amounts of SEPS, the morphology of which is unaffected by annealing.

In Figure 3 the first and the second line correspond to microtomes of the nanocomposites stained by $\mathrm{OsO} 4$ and $\mathrm{RuO} 4$, respectively. On the $\mathrm{RuO} 4$ stained images, bright unstained areas corresponding to PP and dark stained dots corresponding to the styrene blocks of SEPS can be seen. On the other hand, on the $\mathrm{OsO} 4$ stained images, the dark dots correspond to the residual carbon-carbon double bonds 


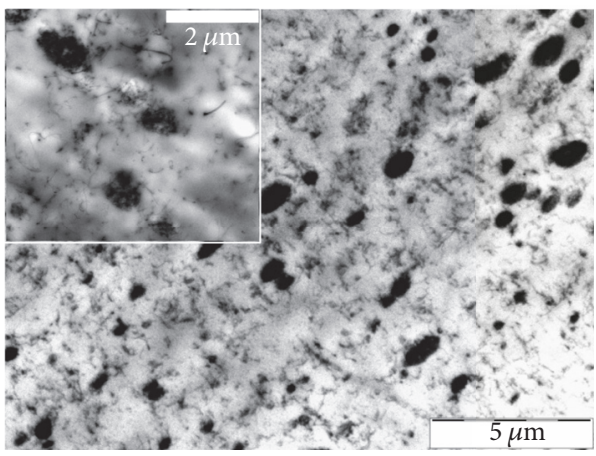

(a)

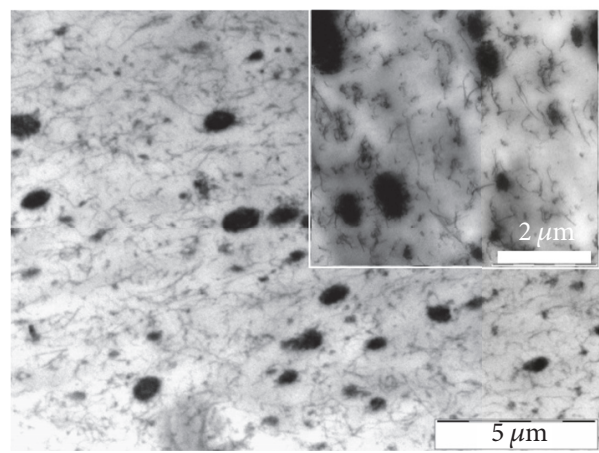

(b)

Figure 1: TEM micrographs of RuO4 stained 2\% CNT PP93 nanocomposites. (a) 1 min annealing; (b) 10 min annealing (see Table 1 for samples compositions).

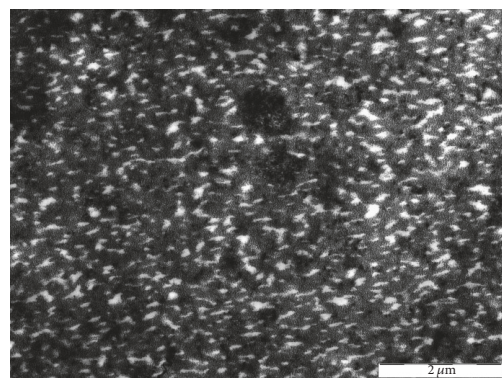

(a)

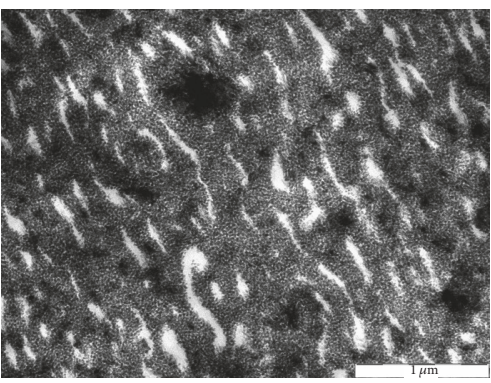

(b)

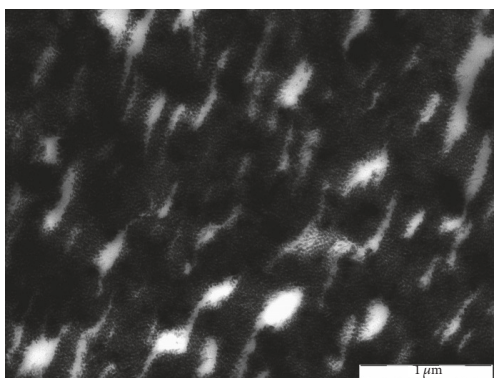

(c)

FIgURE 2: TEM micrograph of RuO4 stained PP50 nanocomposite; (a) 1 min annealing time; (b) 10 min annealing time; (c) 20 min annealing time.

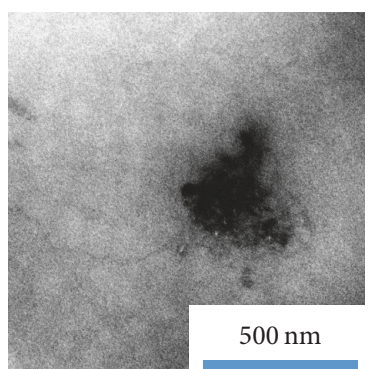

(a)

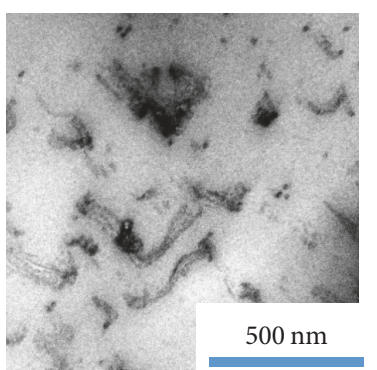

(b)
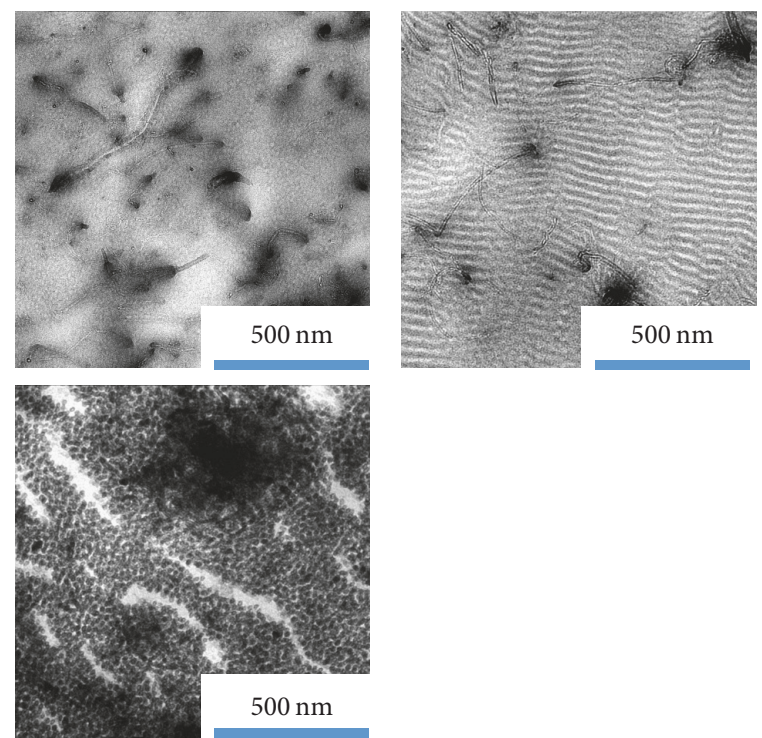

(c) (d)

FIGURE 3: TEM micrographs of stained 2\% CNT nanocomposites. (a): PP98 (1 min annealing); (b) PP93 (1 min annealing); (c) PP50; (d) PP0 (see Table 1 for samples compositions). First line: $\mathrm{OsO} 4$ staining and second line: $\mathrm{RuO} 4$ staining. 


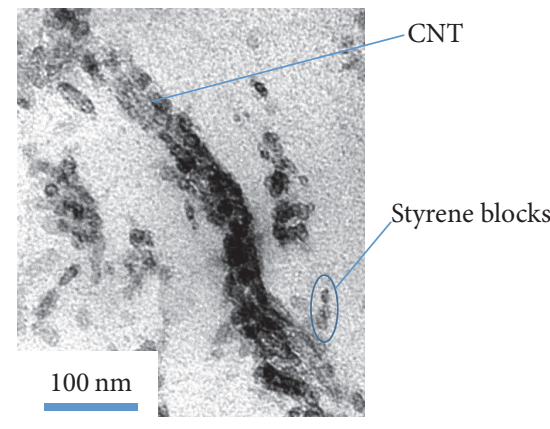

FIGURE 4: High enlargement TEM micrograph of RuO4 stained PP93 composite. Styrene blocks of the SEPS phase (dark dots) aligned along the CNT (dark lines), showing mutual affinity.

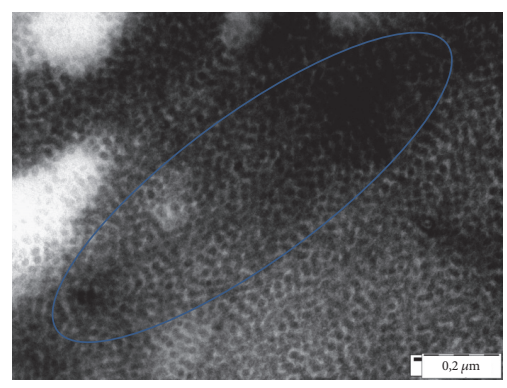

(a)

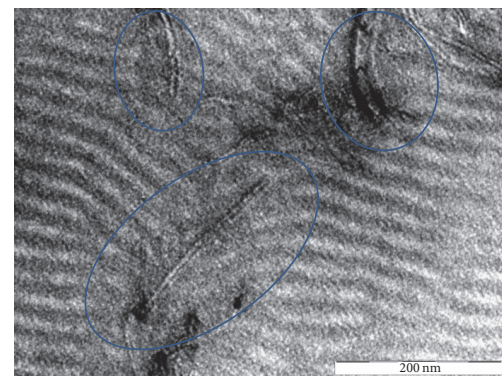

(b)

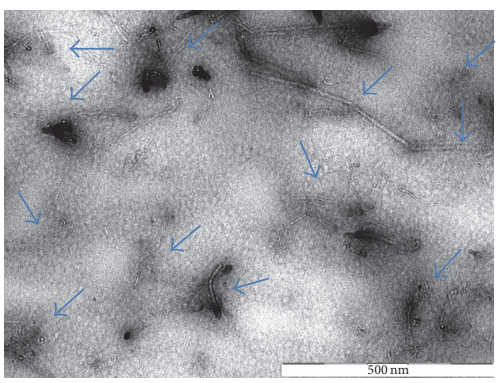

(c)

FIGURE 5: TEM micrograph of (a) PP50 after 10 min annealing time stained with RuO4; (b) PP0 stained with OsO4; (c) PP50 after 10 min annealing time stained with $\mathrm{OsO}$ 4. See main text for further explanations.

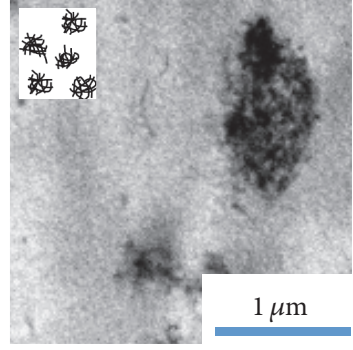

(a)

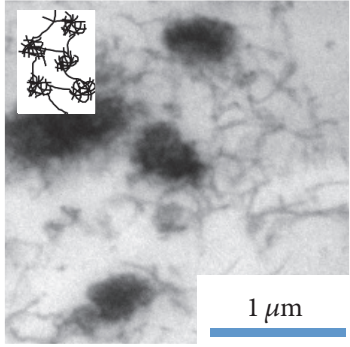

(b)

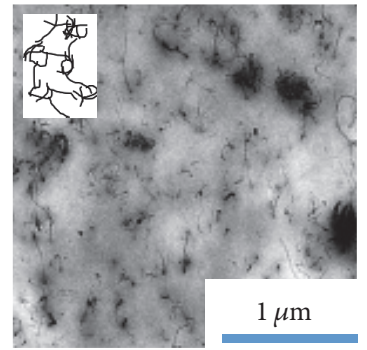

(c)

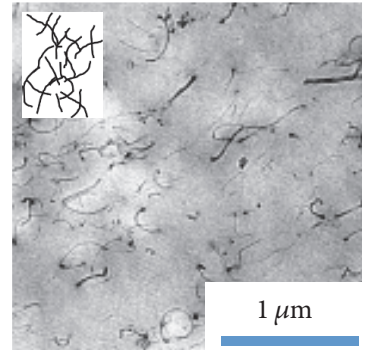

(d)

FIGURE 6: TEM micrographs of RuO4 stained 2\% CNT nanocomposite samples. (a) PP98; (b) PP93; (c) PP50; (d) PP0 (see Table 1 for samples compositions).

in the EP phase of SEPS while the other phases ( $S$ in SEPS and $\mathrm{PP}$ ) remain unstained. The images first show the good overall compatibility of SEPS with PP (see Figures 3(b) and 3(c)). This is due to the presence of EP blocks in SEPS, which are compatible with polyolefins, in particular PP. Second, Figure 3 highlights the very favorable influence of SEPS on the dispersion of CNT in PP. In pure PP (Figure 3(a)), the CNT form big lumps. They are impossible to disperse correctly due to their strong mutual attraction and initial entangled state (from synthesis). Addition of a small quantity of SEPS (5\%, see Figure 3(b)) dramatically improves CNT dispersion, which remains of course excellent also at higher SEPS concentrations (Figures 3(c) and 3(d)).
The strong affinity of the styrene blocks of SEPS for the CNT is confirmed in Figure 4 and is consistent with literature results [24]. At very high SEPS concentration, the styrene blocks entirely cover the CNT and tend to separate them, hence acting as an insulating interlayer.

The detrimental influence of high SEPS concentration can be inferred from the analysis of Figure 5. Indeed, when PP50 is stained by RuO4 (Figure 5(a)) strong darkening around the CNT indicates the presence of SEPS styrene blocks surrounding the nanotubes. Similarly, when PP50 is stained by OsO4, a light grey halo around the CNT reveals SEPS is entirely coating the CNT (Figure 5(c)). Hence, in this sample, the nanotubes are physically separated, which 
is good from the standpoint of CNT dispersion but bad for conductivity. Such halo is absent in PP0 (Figure 5(b)), confirming it is due to SEPS coating the nanotubes.

Figure 6 shows the same systems at a smaller magnification to highlight the overall state of dispersion of the CNT. The chosen staining procedure has been optimized for optimal clarity of the structure imaging. Cartoon insets clarify the evolution from separate lumps in pure PP to fully individualized CNT in pure SEPS with a clear evolution at intermediate PP/SEPS ratios, that is, tight clusters linked by loose connections at 5\% SEPS and loose clusters with extensive connections in the $48 \%$ SEPS case.

\subsection{AC Dielectric Constant and Conductivity PP/SEPS} Nanocomposites with 2 wt\% CNT. At constant CNT loading in the nanocomposite, the balance between CNT agglomerates and the network of well dispersed CNT, and their respective arrangement, modifies the electrical conductivity and dielectric constant. Figures 7 and 8 show the conductivity and dielectric constant values obtained for the reference samples and nanocomposites annealed for $10 \mathrm{~min}$. Although there is a frequency gap between the ranges covered by the two measurement methods, the results are very consistent as the curves from one range can be smoothly extrapolated to the other range. The conductivity and dielectric constant for the reference samples (darker curves) are lower than the corresponding values for the composites. In a pure dielectric polymer, the AC conductivity is mainly due to molecular relaxation at high frequency and DC conductivity is vanishingly small. The PP98, PP93, PP50, and PP0 composites (samples are referenced by their PP content at constant 2\% CNT loading, see Experimental Part) have a conductivity comprised between $10^{-13} \mathrm{~S} / \mathrm{m}$ and $1 \mathrm{~S} / \mathrm{m}$ in the explored frequency range, while the dielectric constant value ranges from 3 to 1000 . A conductivity plateau appears at low frequency for the PP98 and PP95 samples, evolving to a power law (straight line in double log representation) at higher frequencies, with a transition zone in between. For the other two composite samples (PP50 and PP0), a power law is observed over the entire frequency range. The highest conductivity and dielectric constants are obtained at 5\% SEPS (PP93) whereas either high PP (PP98) or high SEPS (PP50, SEPS98) content is less favorable. The differences are the most spectacular at low frequencies.

When SEPS is absent (PP98) or poorly dispersed (large agglomerates of SEPS inside PP are observed in PP50 after only $1 \mathrm{~min}$ annealing), some micrometric agglomerates of $\mathrm{CNT}$ are always present.

\section{Discussion}

The great challenge of correlating dielectric spectroscopy measurements with TEM micrographs is caused by the very different representative volumes of the two methods. Indeed, dielectric measurements give information about a volume around $0.1 \mathrm{~cm}^{3}$ (global information) versus only a few $\mu \mathrm{m}^{3}$ for TEM images (very local information). The dispersion and the percolation threshold can however be linked through the correlation between microscopy observations and measured electrical parameters (electrical conductivity and dielectric constant). The dielectric constant and the AC conductivity depend upon different processes: fluctuations of the dipoles, the propagation of mobile carriers (electrons, holes, or ions diffusion), and the separation of charges at the interfaces. This last mechanism leads to an additional polarization which takes place at inner boundary layers (Maxwell-WagnerSillars polarization). This contribution can be larger than the dielectric response due to molecular fluctuations [49]. Whereas CNT are lumped as big separate agglomerates in pure PP, a small SEPS concentration of 5\% strongly enhances their dispersion and a network between the CNT agglomerates starts to appear (Figures 6(a)-6(c)). When the SEPS concentration is further increased, the strong affinity between the SEPS and the CNT results in a layer of insulating macromolecules all around the nanoparticles (see Figure 4), which partially destroys electrical contacts between them and hence decreases conductivity (Figure 6(d)). To support this interpretation of the experimental results, the conduction mechanism can be represented by the schematic and corresponding equivalent electrical circuit illustrated in Figure 9. Low frequency conduction arises from the network of CNT that are in direct contact. Conductivity is further enhanced at higher frequencies, thanks to capacitive coupling between nontouching CNT agglomerates. The different structures can be modeled as the electrical components of an equivalent electrical circuit. The fluctuations of the molecular dipoles in the hosting polymer matrix correspond to a capacitance; the propagation of the mobile carriers and the separation of the charges at the interface can be represented by different values of a resistance. At low frequency, high conductivity can result from low resistivity CNT agglomerates, provided that they are linked by a sufficient percolating network path.

The equivalent circuit has the following impedance:

$$
\begin{aligned}
Z= & A\left(R_{\text {agglo }}+\frac{1}{j \cdot \omega C_{\mathrm{CNT}}}\right)^{-1}+\frac{B}{R_{\text {network }}} \\
& +\frac{C}{\left(j \omega C_{\text {dipolar }}\right)}, \\
C_{\text {dipolar }}= & \Re\left(C_{\text {dipolar }}\right)(1-j \tan \delta),
\end{aligned}
$$

where $R_{\mathrm{CNT}}$ is the CNT resistance, $C_{\mathrm{CNT}}$ is the capacitance existing between closely adjacent but isolated/nontouching CNT conductive agglomerates, $\mathfrak{R}\left(C_{\text {dipolar }}\right)$ is the capacitance associated with molecular fluctuations/molecular dipoles in the hosting polymer matrix, and $\tan \delta$ is its loss tangent factor while $R_{\text {network }}$ is the resistance accounting for the conductive network existing inside the polymer matrix due to existing physical contacts between conductive fillers: CNT having resistance $R_{\mathrm{CNT}}$ either interconnected or creating conductive paths between isolated agglomerates having resistance $R_{\text {agglo }}$. The dielectric polarization losses of the polymer matrix can have a significant influence and are accounted for by an imaginary part of the equivalent dipolar capacitance, while factors $A$ and $B$ account for the relative volumetric fractions of interconnected and isolated CNT agglomerates in the 


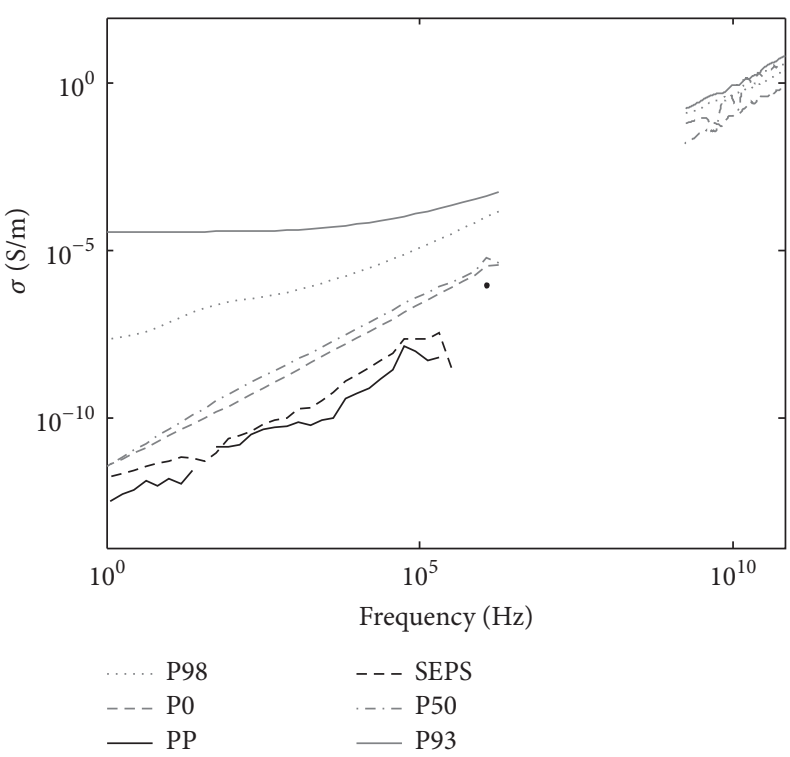

(a)

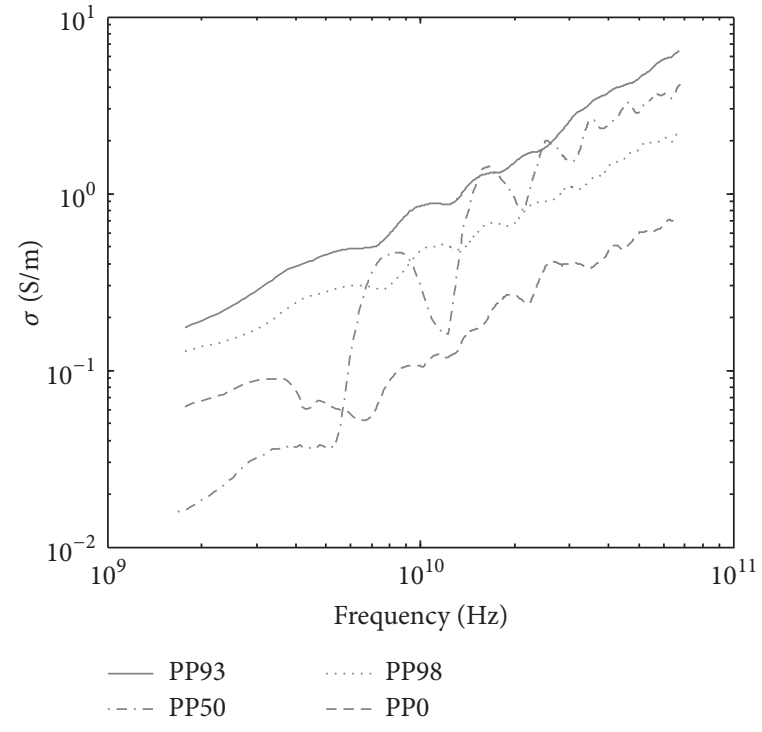

(b)

FIGURE 7: (a) Electrical conductivity in the $1 \mathrm{~Hz}-67 \mathrm{GHz}$ frequency range. The lowest conductivities are measured for the pure SEPS and PP matrices. With $2 \%$ wt CNT, the conductivities range from $10^{-13}$ to $10^{-4} \mathrm{~S} / \mathrm{m}$ at low frequencies, depending on PP content. The highest conductivity is obtained at 5\% SEPS (PP93). (b) Electrical conductivity in the $1.7 \mathrm{GHz}-67 \mathrm{GHz}$ frequency range.

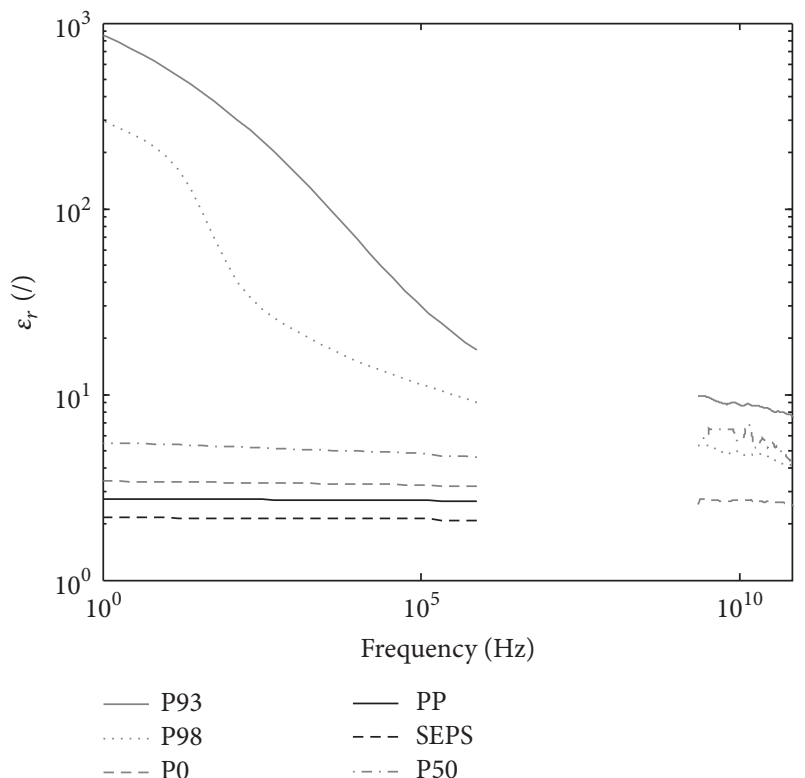

Figure 8: Dielectric constant in the $1 \mathrm{~Hz}-67 \mathrm{GHz}$ frequency range. Highest values correspond to samples having highest conductivity in Figure 7.

hosting matrix. Predictions of the frequency dependence of the conductivity using the electrical circuit model are shown in Figure 10 for two limiting cases of well dispersed CNT and large agglomerates, respectively. The model's parameters are given in Table 2.

The low frequency measurements are highly dependent on the resistive component of the composite: the resistance
TABLE 2: Model's parameters.

\begin{tabular}{lcc}
\hline & Good dispersion & CNT agglomerates \\
\hline $\mathrm{A}$ & 0.5 & 1 \\
$\mathrm{~B}$ & 0.5 & 0.6 \\
$\mathrm{C}$ & -0.5 & -0.5 \\
$\tan \delta$ & 0.0011 & 0.005 \\
$R_{\text {agglo }}$ & $0.01 \Omega$ & $10^{-8} \Omega$ \\
$C_{\mathrm{CNT}}$ & $10^{-12}-i 10^{-15} \mathrm{~F}$ & $10^{-9}-i 5 \cdot 10^{-12} \mathrm{~F}$ \\
$R_{\text {network }}$ & $10^{12} \Omega$ & $10^{-8} \Omega$ \\
$C_{\text {dipolar }}$ & $10^{-10}-i 10^{-13} \mathrm{~F}$ & $10^{-7}-i 5 \cdot 10^{-10} \mathrm{~F}$ \\
\hline
\end{tabular}

is higher for well dispersed CNT (PP50 and PP0) than for networks containing agglomerates (PP93). This is probably due to higher contact resistance between the nanotubes owing to an adsorbed insulating SEPS layer (see Figure 4). On the other hand, the high frequency behavior mainly depends on capacitive coupling and follows a power law versus frequency, independently of the microstructure, but the structure of the network and the CNT dispersion strongly influences the conductivity close to the percolation rate. The physics of the material is mainly related to the different properties of each component and the internal structure of the composite. For higher rates of CNT, the network looks like a global agglomerate of entangled CNT. And the current can then pass across many paths, increasing the electrical conductivity and the crossover frequency. At lower rates of $\mathrm{CNT}$, the number of conductive particles is too low to form an electrical network, independently of the network structure, and then the composite is an insulator material. 

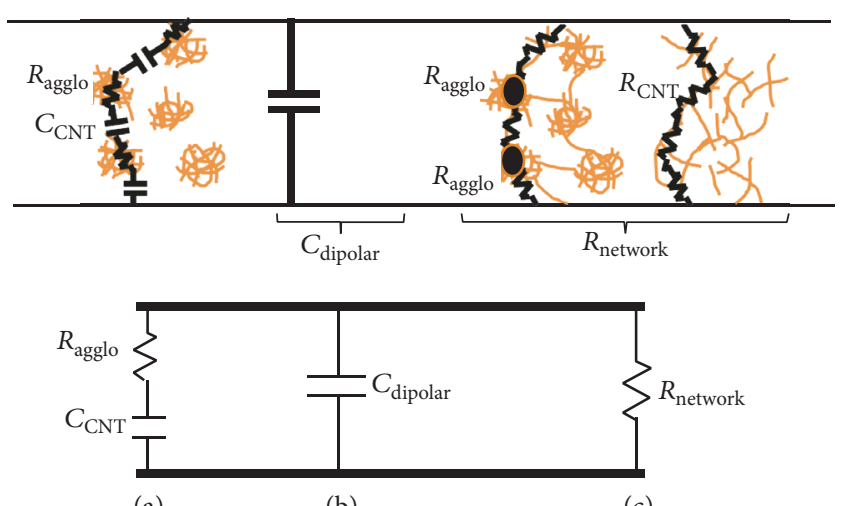

(a)

(b)

(c)

FIGURE 9: Schematic of the CNT dispersion mechanisms inside a polymer matrix: CNT agglomerates closely separated by polymer, capacitance associated with dipolar/molecular fluctuations of free/unfilled polymer areas, and DC/low frequency conduction paths mechanisms: agglomerates connected by isolated CNT, and network of interconnected well dispersed CNT. Corresponding equivalent circuit includes (a) a resistance and a capacitance representing the closely spaced agglomerates and their capacitive coupling through polymer separating them, (b) a capacitance associated with dipolar motion, and (c) a resistance modeling the network of interconnected conductive fillers (agglomerates and/or dispersed CNT).

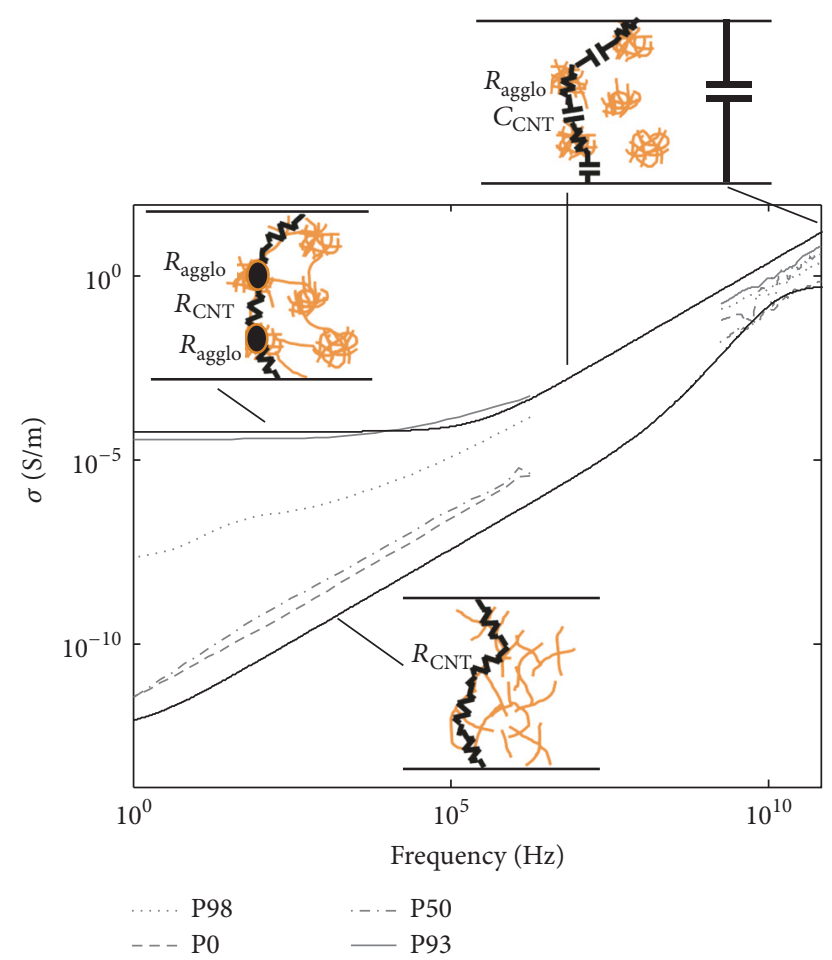

FIGURE 10: Predictions (solid black lines) of the frequency dependence of the conductivity using the electrical circuit model shown in Figure 9 for two limiting cases of well dispersed CNT and large agglomerates, respectively. Model's parameters corresponding to each case are given in Table 2.

\section{Absorption}

The electrical properties influence the electromagnetic absorption. The samples PP0 and PP50 have a low dielectric constant independent of the frequency; and the electrical conductivity shows a power law behavior as a function of the frequency. The samples PP0 and PP93 show a different behavior at low frequency: the dielectric constant and electrical conductivity dramatically increase below $100 \mathrm{kHz}$. This behavior directly impacts the absorption: the coupled values (electrical conductivity and dielectric constant) for an optimal absorption depend consequently on the frequency; hence the best material for absorption changes if the frequency is modified. The absorption can be estimated as a function of the dielectric constant and conductivity, frequency, and thickness of the sample, according to the formalism described in $[48,50]$.

6.1. $1 \mathrm{~Hz}-100 \mathrm{MHz}$ Frequency Range. On Figure 11 the absorption levels are very low (less than $1 \%$ ). The absorption is globally higher for PP93 and increases for frequencies above $100 \mathrm{MHz}$. The absorption of PP98 also increases above $100 \mathrm{kHz}$. The reflection decreases due to the reduction of the dielectric constant while the transmission decreases due to the increase of the electrical conductivity resulting in an increase of the absorption.

6.2. $100 \mathrm{MHz}-67 \mathrm{GHz}$. The electrical conductivities show a power law behavior and are almost parallel. The range goes from PP0 to PP98 and to PP93 (which has the highest conductivity). The sample PP50 has a particular behavior and thus is not included in the first step of comparison. The high values of conductivity imply a significant reduction of the transmission factor which radically decreases the transmission level and impacts also the reflection level (less significative increase). Figure 12 shows the absorption rate as a function of frequency for sample thickness of $2 \mathrm{~mm}$. The optimal couple is 3-0.01 to $10 \mathrm{~S} / \mathrm{m}$. The sample PP93 is the best material for this frequency range. Increasing the frequency does not influence the order of materials having the best absorption for this thickness (PP0, PP98, and PP93) which is the same as the measured conductivity.

\section{Conclusion}

We have studied the link between the dispersion of multiwall CNT inside a melt compounded PP matrix and the corresponding electrical properties over a broad frequency range as a function of melt annealing time and the concentration of an SEPS block copolymer compatibilizer. The annealing time is an important process parameter which significantly influences the overall morphology and conductivity for samples around 50/50 PP/SEPS ratio. In such case, melt annealing for $10 \mathrm{~min}$ allows sufficient macromolecular interdiffusion to reach overall morphological and compositional homogeneity. On the other hand, samples containing only a very small concentration of the minor phase are unaffected by annealing. The low as well as high frequency 


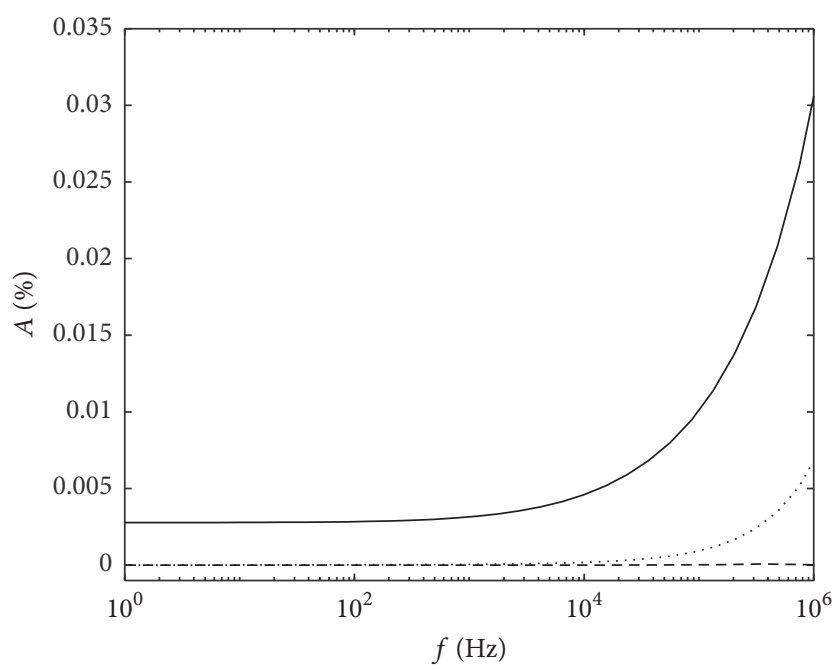

FIGURE 11: Predictions of absorption for thickness of sample $=2 \mathrm{~mm}$, in frequency range $1 \mathrm{~Hz}-100 \mathrm{MHz}$.

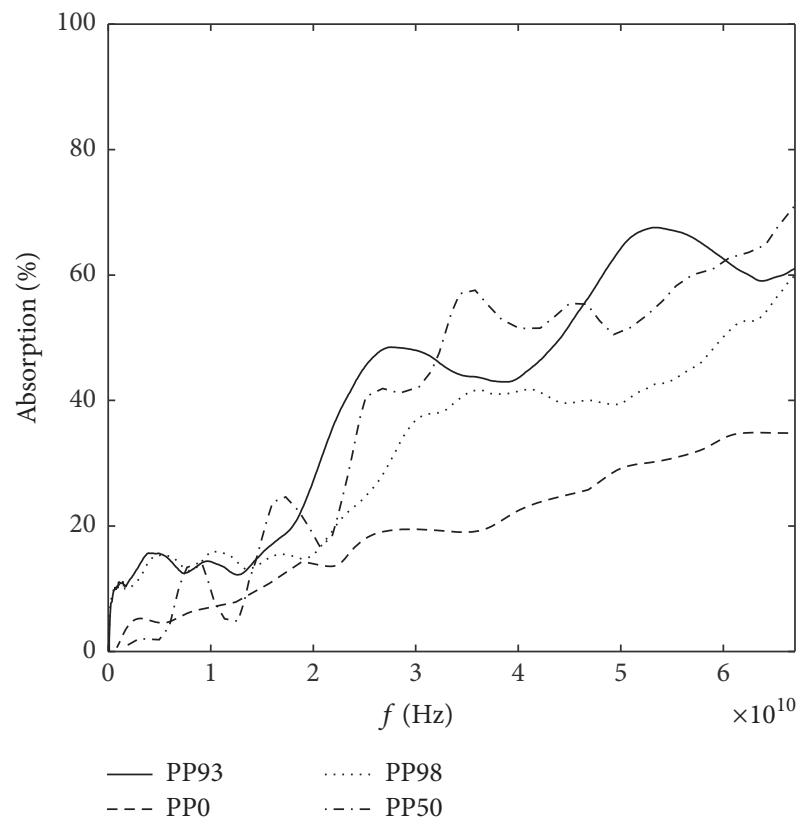

FIGURE 12: Predictions of absorption for thickness of sample $=2 \mathrm{~mm}$, in frequency range $100 \mathrm{MHz}-67 \mathrm{GHz}$.

conductivity reaches an optimum at small concentration of compatibilizer. This is due to a balance between the presence of highly conducting but separate CNT agglomerates and of a conducting path network made of individualized CNT. The balance between the two is controlled by the compatibilizer concentration. If the latter is too low, the CNT agglomerates remain separate and the composite does not conduct. At the opposite, when the compatibilizer concentration is too high, the CNT form an extensive network but the contact resistance between them reduces conductivity due to the presence an insulating adsorbed layer. The schematic structure observed as a function of compatibilizer concentration (after sufficient annealing time) can be semiquantitatively modeled by an equivalent electrical circuit which shows that resistive effects dominate at low frequency while capacitive ones dominate at high frequency.

\section{Conflicts of Interest}

The authors declare that there are no conflicts of interest regarding the publication of this paper.

\section{Acknowledgments}

The support of Pascal Simon for electrical measurements and of Isabel Molenberg for fruitful discussions is gratefully acknowledged. The authors are also grateful for the "Communauté Française de Belgique" through its research program "Actions de Recherches Concertées: Project Nano4waves" and for the National Fund for Scientific Research (F.R.S.-FNRS, Belgium) for financial support.

\section{References}

[1] I. Alig, P. Pötschke, D. Lellinger et al., "Establishment, morphology and properties of carbon nanotube networks in polymer melts," Polymer, vol. 53, no. 1, pp. 4-28, 2012.

[2] J. N. Coleman, U. Khan, W. J. Blau, and Y. K. Gun'ko, "Small but strong: a review of the mechanical properties of carbon nanotube-polymer composites," Carbon, vol. 44, no. 9, pp. 16241652,2006

[3] J. N. Coleman, U. Khan, and Y. K. Gun'ko, "Mechanical reinforcement of polymers using carbon nanotubes," Advanced Materials, vol. 18, no. 6, pp. 689-706, 2006.

[4] E. Logakis, C. H. Pandis, P. Pissis, J. Pionteck, and P. Pötschke, "Highly conducting poly(methyl methacrylate)/carbon nanotubes composites: Investigation on their thermal, dynamicmechanical, electrical and dielectric properties," Composites Science and Technology, vol. 71, no. 6, pp. 854-862, 2011.

[5] P. Pötschke, S. M. Dudkin, and I. Alig, "Dielectric spectroscopy on melt processed polycarbonate-multiwalled carbon nanotube composites," Polymer, vol. 44, no. 17, pp. 5023-5030, 2003.

[6] S. Rul, F. Lefèvre-Schlick, E. Capria, C. Laurent, and A. Peigney, "Percolation of single-walled carbon nanotubes in ceramic matrix nanocomposites," Acta Materialia, vol. 52, no. 4, pp. 1061-1067, 2004.

[7] S. Barrau, P. Demont, A. Peigney, C. Laurent, and C. Lacabanne, "Dc and ac conductivity of carbon nanotubes-polyepoxy composites," Macromolecules, vol. 36, no. 14, pp. 5187-5194, 2003.

[8] Y. He, H. Deng, X. Jiao, S. He, J. Gao, and X. Yang, "Infrared perfect absorber based on nanowire metamaterial cavities," Optics Letters, vol. 38, no. 7, pp. 1179-1181, 2013.

[9] V. Sunny, P. Kurian, P. Mohanan, P. A. Joy, and M. R. Anantharaman, "A flexible microwave absorber based on nickel ferrite nanocomposite," Journal of Alloys and Compounds, vol. 489, no. 1, pp. 297-303, 2010.

[10] Y. Yang, C. Xu, Y. Xia, T. Wang, and F. Li, "Synthesis and microwave absorption properties of FeCo nanoplates," Journal of Alloys and Compounds, vol. 493, no. 1-2, pp. 549-552, 2010.

[11] S. P. Gairola, V. Verma, A. Singh, L. P. Purohit, and R. K. Kotnala, "Modified composition of barium ferrite to act as a microwave 
absorber in X-band frequencies," Solid State Communications, vol. 150, no. 3-4, pp. 147-151, 2010.

[12] R. S. Meena, S. Bhattachrya, and R. Chatterjee, "Development of "tuned microwave absorbers" using U-type hexaferrite," Materials and Design, vol. 31, no. 7, pp. 3220-3226, 2010.

[13] T.-H. Ting and K.-H. Wu, "Synthesis, characterization of polyaniline/BaFe12O19 composites with microwave-absorbing properties," Journal of Magnetism and Magnetic Materials, vol. 322, no. 15, pp. 2160-2166, 2010.

[14] K.-Y. Park, J.-H. Han, S.-B. Lee, J.-B. Kim, J.-W. Yi, and S.-K. Lee, "Fabrication and electromagnetic characteristics of microwave absorbers containing carbon nanofibers and NiFe particles," Composites Science and Technology, vol. 69, no. 7-8, pp. 12711278, 2009.

[15] I. M. De Rosa, F. Sarasini, M. S. Sarto, and A. Tamburrano, "EMC impact of advanced carbon fiber/carbon nanotube reinforced composites for next-generation aerospace applications," IEEE Transactions on Electromagnetic Compatibility, vol. 50, no. 3, pp. 556-563, 2008.

[16] Y. J. Kim, K. J. An, K. S. Suh et al., "Hybridization of oxidized MWNT and silver powder in polyurethane matrix for electromagnetic interference shielding application," IEEE Transactions on Electromagnetic Compatibility, vol. 47, no. 4, pp. 872-879, 2005.

[17] C. P. Neo and V. K. Varadan, "Optimization of carbon fiber composite for microwave absorber," IEEE Transactions on Electromagnetic Compatibility, vol. 46, no. 1, pp. 102-106, 2004.

[18] D. Micheli, R. Pastore, C. Apollo et al., "Broadband electromagnetic absorbers using carbon nanostructure-based composites," IEEE Transactions on Microwave Theory and Techniques, vol. 59, no. 10, pp. 2633-26646, 2011.

[19] K. Lakshmi, H. John, K. T. Mathew, R. Joseph, and K. E. George, "Microwave absorption, reflection and EMI shielding of PUPANI composite," Acta Materialia, vol. 57, no. 2, pp. 371-375, 2009.

[20] Y. Yang, M. C. Gupta, K. L. Dudley, and R. W. Lawrence, "Novel carbon nanotube-polystyrene foam composites for electromagnetic interference shielding," Nano Letters, vol. 5, no. 11, pp. 21312134, 2005.

[21] J.-M. Thomassin, C. Jérôme, T. Pardoen, C. Bailly, I. Huynen, and C. Detrembleur, "Polymer/carbon based composites as electromagnetic interference (EMI) shielding materials," Materials Science and Engineering R: Reports, vol. 74, no. 7, pp. 211232, 2013.

[22] M. Arjmand, T. Apperley, M. Okoniewski, and U. Sundararaj, "Comparative study of electromagnetic interference shielding properties of injection molded versus compression molded multi-walled carbon nanotube/polystyrene composites," Carbon, vol. 50, no. 14, pp. 5126-5134, 2012.

[23] V. Panwar, J.-O. Park, S.-H. Park, S. Kumar, and R. M. Mehra, "Electrical, dielectric, and electromagnetic shielding properties of polypropylene-graphite composites," Journal of Applied Polymer Science, vol. 115, no. 3, pp. 1305-1314, 2010.

[24] F. Tao, L. Bonnaud, O. Murariu, D. Auhl, P. Dubois, and C. Bailly, "A convenient route to high-performance HDPE-CNT conductive nanocomposites by control of matrix nucleation," Macromolecular Chemistry and Physics, vol. 213, no. 21, pp. 2275-2281, 2012.

[25] D. P. Almond and C. R. Bowen, "Anomalous power law dispersions in ac conductivity and permittivity shown to be characteristics of microstructural electrical networks," Physical Review Letters, vol. 92, no. 15, pp. 157601-1, 2004.
[26] C. R. Bowen and D. P. Almond, "Modelling the 'universal' dielectric response in heterogeneous materials using microstructural electrical networks," Materials Science and Technology, vol. 22, no. 6, pp. 719-724, 2006.

[27] P. Richardson, D. P. Almond, and C. R. Bowen, "A study of random capacitor networks to assess the emergent properties of dielectric composites," Ferroelectrics, vol. 391, no. 1, pp. 158-167, 2009.

[28] M. Mahmoodi, M. Arjmand, U. Sundararaj, and S. Park, "The electrical conductivity and electromagnetic interference shielding of injection molded multi-walled carbon nanotube/polystyrene composites," Carbon, vol. 50, no. 4, pp. 14551464, 2012.

[29] Y. Yang, M. C. Gupta, and K. L. Dudley, “Towards costefficient EMI shielding materials using carbon nanostructurebased nanocomposites," Nanotechnology, vol. 18, no. 34, Article ID 345701, 2007.

[30] G. A. Gelves, M. H. Al-Saleh, and U. Sundararaj, "Highly electrically conductive and high performance EMI shielding nanowire/polymer nanocomposites by miscible mixing and precipitation," Journal of Materials Chemistry, vol. 21, no. 3, pp. 829-836, 2011.

[31] M. Arjmand, M. Mahmoodi, G. A. Gelves, S. Park, and U. Sundararaj, "Electrical and electromagnetic interference shielding properties of flow-induced oriented carbon nanotubes in polycarbonate," Carbon, vol. 49, no. 11, pp. 3430-3440, 2011.

[32] A. P. Singh, B. K. Gupta, M. Mishra, A. Chandra, R. B. Mathur, and S. K. Dhawan, "Multiwalled carbon nanotube/cement composites with exceptional electromagnetic interference shielding properties," Carbon, vol. 56, pp. 86-96, 2013.

[33] A. Saib, L. Bednarz, R. Daussin et al., "Carbon nanotube composites for broadband microwave absorbing materials," IEEE Transactions on Microwave Theory and Techniques, vol. 54, no. 6, pp. 2745-2753, 2006.

[34] P. Verge, S. Benali, L. Bonnaud et al., "Unpredictable dispersion states of MWNTs in HDPE: A comparative and comprehensive study," European Polymer Journal, vol. 48, no. 4, pp. 677-683, 2012.

[35] F. Tao, B. Nysten, A.-C. Baudouin et al., "Influence of nanoparticle-polymer interactions on the apparent migration behaviour of carbon nanotubes in an immiscible polymer blend," Polymer, vol. 52, no. 21, pp. 4798-4805, 2011.

[36] G. S. Nanda, R. Sravendra, W. C. Jae, L. Lin, and H. C. Siew, "Polymer nanocomposites based on functionalized carbon nanotubes," Progress in Polymer Science, vol. 35, no. 7, pp. 837$867,2010$.

[37] F. Tao, L. Bonnaud, D. Auhl, B. Struth, P. Dubois, and C. Bailly, "Influence of shear-induced crystallization on the electrical conductivity of high density polyethylene carbon nanotube nanocomposites," Polymer (United Kingdom), vol. 53, no. 25, pp. 5909-5916, 2012.

[38] H. Deng, T. Skipa, R. Zhang et al., "Effect of melting and crystallization on the conductive network in conductive polymer composites," Polymer, vol. 50, no. 15, pp. 3747-3754, 2009.

[39] K. Menzer, B. Krause, R. Boldt, B. Kretzschmar, R. Weidisch, and P. Pötschke, "Percolation behaviour of multiwalled carbon nanotubes of altered length and primary agglomerate morphology in melt mixed isotactic polypropylene-based composites," Composites Science and Technology, vol. 71, no. 16, pp. 1936-1943, 2011. 
[40] M. Micusík, M. Omastova, I. Krupa et al., "A comparative study on the Electrical and mechanical behaviour of multiwalled carbon nanotube composites prepared by diluting a masterbatch with various types of polypropylenes," Journal of Applied Polymer Science, vol. 113, no. 4, pp. 2536-2551, 2009.

[41] A.-C. Baudouin, J. Devaux, and C. Bailly, "Localization of carbon nanotubes at the interface in blends of polyamide and ethylene-acrylate copolymer," Polymer, vol. 51, no. 6, pp. 13411354, 2010.

[42] A.-C. Baudouin, C. Bailly, and J. Devaux, "Interface localization of carbon nanotubes in blends of two copolymers," Polymer Degradation and Stability, vol. 95, no. 3, pp. 389-398, 2010.

[43] A.-C. Baudouin, D. Auhl, F. Tao, J. Devaux, and C. Bailly, "Polymer blend emulsion stabilization using carbon nanotubes interfacial confinement," Polymer, vol. 52, no. 1, pp. 149-156, 2011.

[44] N. C. Das, Y. Liu, K. Yang, W. Peng, S. Maiti, and H. Wang, "Single-walled carbon nanotube/poly(methyl methacrylate) composites for electromagnetic interference shielding," Polymer Engineering and Science, vol. 49, no. 8, pp. 1627-1634, 2009.

[45] J. A. King, W. A. Pisani, D. R. Klimek-Mcdonald, W. F. Perger, G. M. Odegard, and D. G. Turpeinen, "Shielding effectiveness of carbon-filled polypropylene composites," Journal of Composite Materials, vol. 50, no. 16, pp. 2177-2189, 2016.

[46] C.-L. Huang, C.-W. Lou, C.-F. Liu, C.-H. Huang, X.-M. Song, and J.-H. Lin, "Polypropylene/graphene and polypropylene/carbon fiber conductive composites: Mechanical, crystallization and electromagnetic properties," Applied Sciences (Switzerland), vol. 5, no. 4, pp. 1196-1210, 2015.

[47] A. Ameli, P. U. Jung, and C. B. Park, "Electrical properties and electromagnetic interference shielding effectiveness of polypropylene/carbon fiber composite foams," Carbon, vol. 60, pp. 379-391, 2013.

[48] N. Quiévy, P. Bollen, J.-M. Thomassin et al., "Electromagnetic absorption properties of carbon nanotube nanocomposite foam filling honeycomb waveguide structures," IEEE Transactions on Electromagnetic Compatibility, vol. 54, no. 1, pp. 43-51, 2012.

[49] F. Kremer and A. Schönhals, Broadband Dielectric Spectroscopy, Springer, 2003.

[50] I. Huynen, N. Quiévy, C. Bailly et al., "Multifunctional hybrids for electromagnetic absorption," Acta Materialia, vol. 59, no. 8, pp. 3255-3266, 2011. 

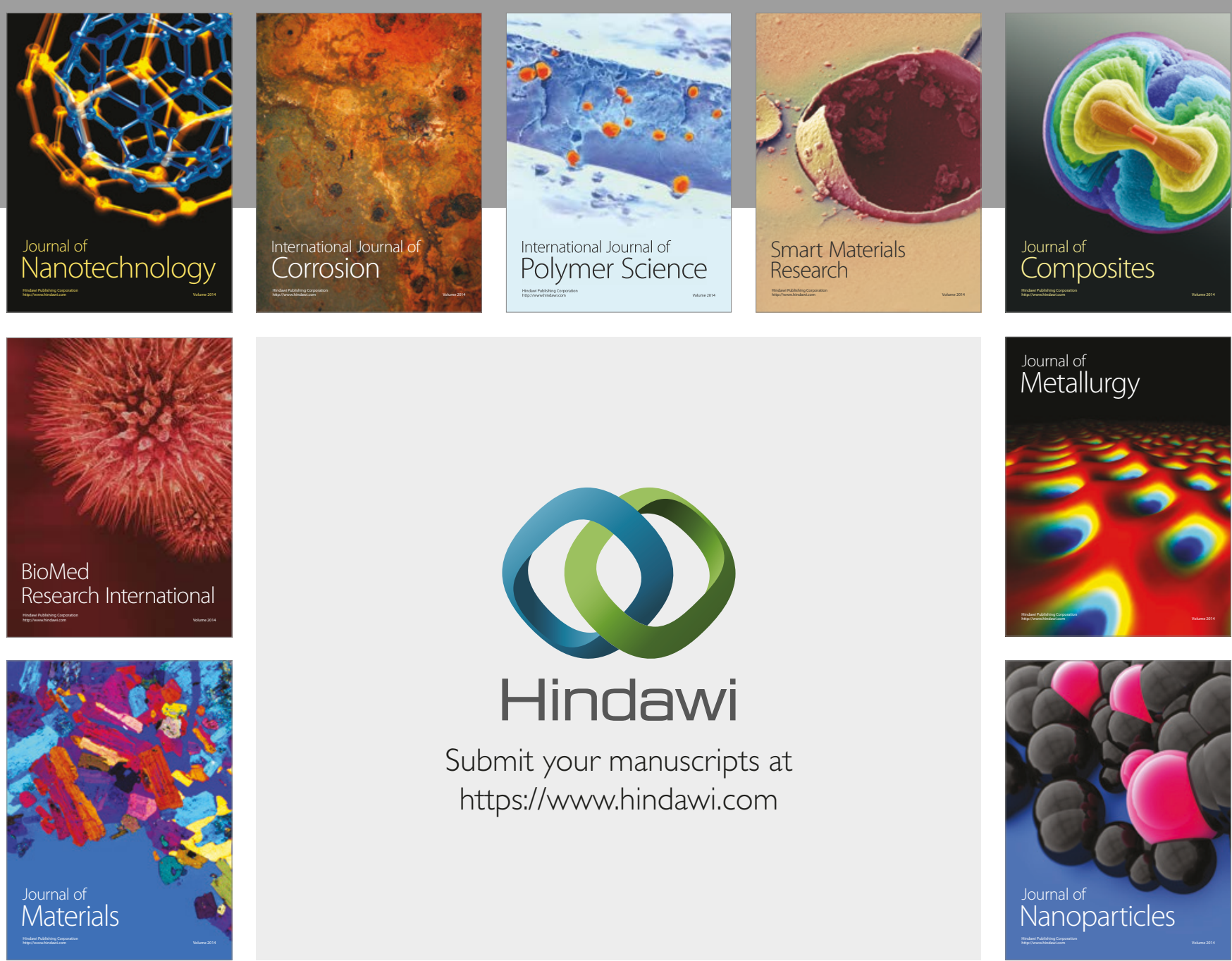

\section{Hindawi}

Submit your manuscripts at

https://www.hindawi.com
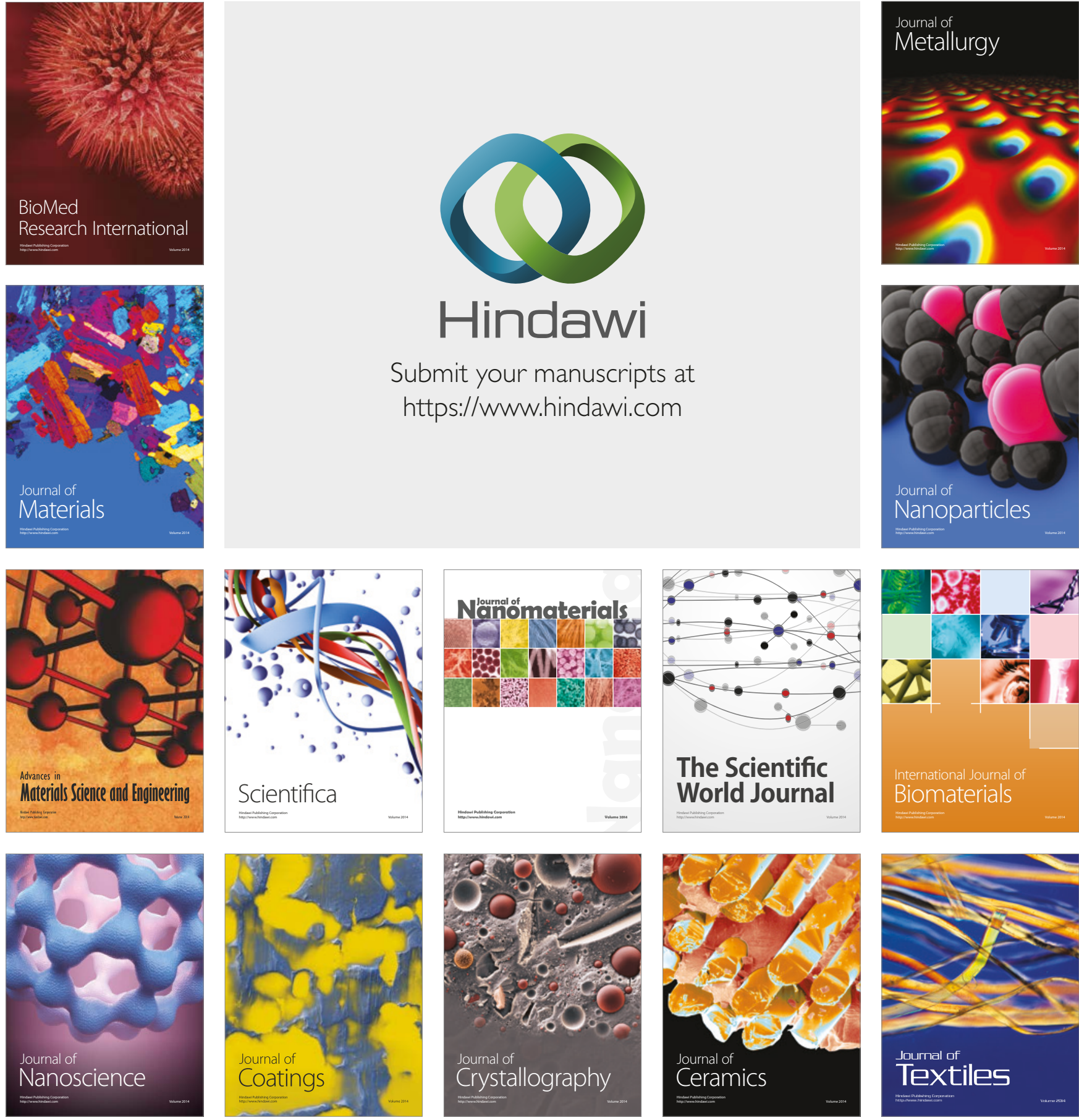

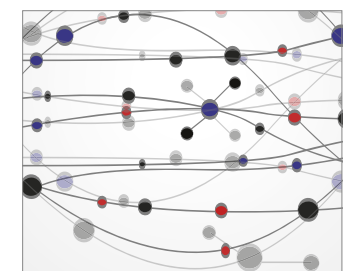

The Scientific World Journal
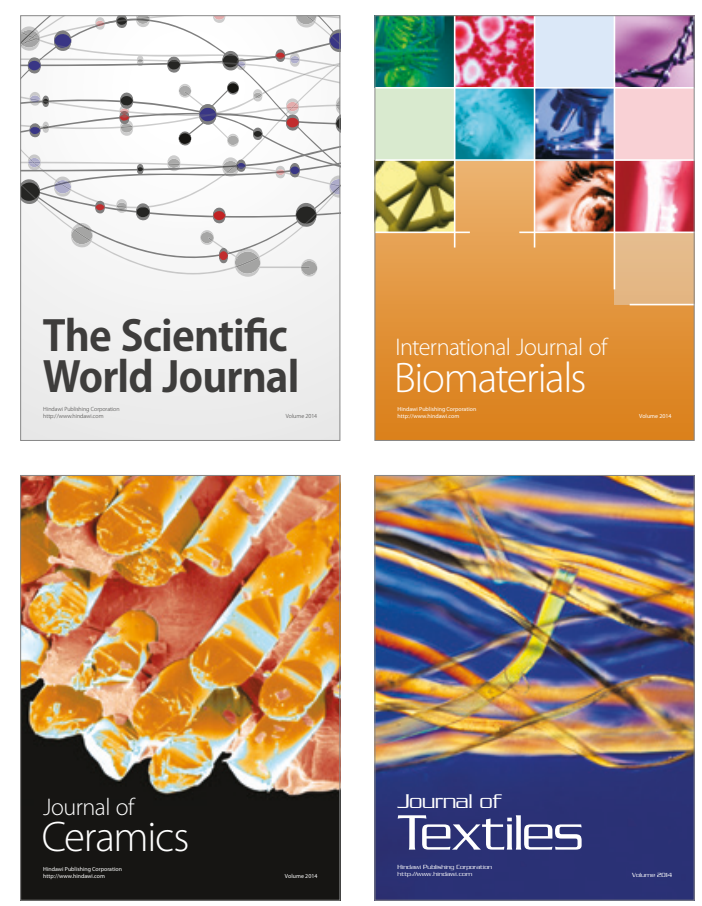